EXTENDED REPORT

\title{
Childhood myopia and parental smoking
}

\author{
S-M Saw, K-S Chia, J M Lindstrom, D T H Tan, R A Stone
}

Br J Ophthalmol 2004;88:934-937. doi: 10.1136/bjo.2003.033175

Aim: To examine the relation between exposure to passive parental smoke and myopia in Chinese children in Singapore.

Methods: 1334 Chinese children from three schools in Singapore were recruited, all of whom were participants in the Singapore Cohort study Of the Risk factors for Myopia (SCORM).

See end of article for authors' affiliations ......................

Correspondence to: Associate Professor S-M Saw, Department of Community, Occupational and Family Medicine, National University of Singapore, 16 Medical Drive, Singapore 117597 , Republic of Singapore; cotsawsm@nus.edu.sg

Accepted for publication 1 October 2003 Information on whether the father or mother smoked, number of years smoked, and the number of cigarettes smoked per day during the child's lifetime were derived. These data were correlated with contemporaneously obtained data available in SCORM. The children's cycloplegic autorefraction, corneal curvature radius, and biometry measures were compared with reported parental smoking history.

Results: There were 434 fathers (33.3\%) and 23 mothers (1.7\%) who smoked during their child's lifetime. There were no significant trends observed between paternal smoking and refractive error or axial length. After controlling for age, sex, school, mother's education, and mother's myopia, children with mothers who had ever smoked during their lifetime had more "positive" refractions (adjusted mean $-0.28 \mathrm{D} v$ -1.38 D) compared with children whose mother did not smoke $(p=0.012)$.

Conclusions: The study found no consistent evidence of association between parental smoking and refractive error. There was a suggestion that children whose mothers smoked cigarettes had more hyperopic refractions, but the absence of a relation with paternal smoking and the small number of mothers who smoked in this sample preclude definite conclusions about a link between passive smoking exposure and myopia.
$\mathrm{D}$ espite extensive research, the aetiology of clinical myopia is poorly understood. Both environmental and genetic factors are presumed to contribute to refractive errors, but defining either the environmental influences or the involved genes in a form that permits biologically based hypotheses has proved elusive. ${ }^{12}$ Recent laboratory research has shown that visual input modulates refractive development, and has implicated a variety of neural and retinal mechanisms in experimental animals, with initial extrapolation to humans. ${ }^{3}$

Muscarinic acetylcholine receptors are among the most extensively studied receptor systems that seemingly influence refractive development. ${ }^{45}$ Nicotinic cholinergic receptors comprise the other main class of acetylcholine receptors. ${ }^{6}$ These ion channel receptors mediate fast neurotransmission at cholinergic synapses of the neuromuscular junction and the nervous system. In chicks, antagonists acting at neural nicotinic acetylcholine receptors inhibit experimental myopia. ${ }^{7}$ Accordingly, it has been hypothesised that neural forms of nicotinic acetylcholine receptors in the retina might have a role in refractive development. Exposure to nicotine in cigarette smoke may activate nicotinic cholinergic receptors and induce shifts in refraction, including the development of myopia. To explore the potential applicability of this hypothesis to humans, we sought an association of refractive development in young children with nicotine exposure through passive smoking by their parents.

\section{METHODS}

Subjects were recruited from the Singapore Cohort study Of the Risk factors for Myopia (SCORM), the methodology of which has been described previously. ${ }^{8-10}$ In brief, children in grades 1 to 3 (aged 7-9 years) were recruited from three schools in Singapore to join this study in 1999 and 2001. Because of logistical and practical constraints, the three schools were not randomly sampled but selected by the Ministry of Education. Children with serious medical conditions such as heart disorders or serious eye conditions such as cataract were excluded. For this cross sectional study, Chinese children with refraction data measurements $(\mathrm{n}=1334)$ from two schools in November 2001 and one school in May 2002 and whose parents completed a questionnaire on smoking habits were included. Only Chinese children were included in this study to facilitate the investigation of the relation between parental smoking and refraction in an ethnically homogenous population. Of the 2192 eligible children, 1485 children agreed to participate and parental smoking data were available for 1334 children. The participation rate in SCORM among students at the three schools was $60.9 \%$ (1334 of 2192). The proportion of children who reported short sightedness before the first eye examination were not different in those who participated in SCORM $(30.7 \%)$ and those who did not $(28.2 \%)(p=0.40)$. The ethics committee of the Singapore Eye Research Institute approved the study, and the University of Pennsylvania Institutional Review Board approved participation in SCORM projects. The conduct of the study followed the tenets of the Declaration of Helsinki.

\section{REFRACTIVE ERROR MEASUREMENTS}

Ocular examinations were conducted as part of ongoing eye examinations of the SCORM study. Cycloplegia was induced in each eye by the administration of $1 \%$ cyclopentolate solution at 5 minute intervals. Thirty minutes after the instillation of the last drop, one of two calibrated autokeratorefractometers (RK5, Canon Inc, Ltd, Tochigiken, Japan) was used to obtain five consecutive readings. Spherical equivalent was defined as sphere +0.5 negative cylinder power. The mean spherical equivalent refraction of the average of the five

Abbreviations: SCORM, Singapore Cohort study of the Risk factors for Myopia 
readings was used. The average of the corneal curvature readings along the flatter and steeper meridians was obtained. The average of six axial lengths, anterior chamber depth, lens thickness, and vitreous chamber depth readings were obtained using one of two calibrated biometry ultrasound units (probe frequency of $10 \mathrm{mHz}$; Echoscan US-800, Nidek Co Ltd, Tokyo). If the standard deviation (SD) of the six measurements was $0.12 \mathrm{~mm}$ or greater, the data were not included and the measurements repeated until the SD was less than $0.12 \mathrm{~mm}$. Biometry data could not be performed for 12 children as they were not cooperative. The personnel were masked to parental smoking information when the ocular measurements were made.

\section{PARENTAL SMOKING INFORMATION}

The parents completed a questionnaire on their smoking habits. Questions included whether the father or mother were current or former cigarette smokers (defined as at least one cigarette per day for one year or longer). Data on whether the father or mother smoked during the child's lifetime were calculated from the initiation and cessation dates reported of parental smoking. The number of cigarettes each parent smoked during the child's lifetime was also estimated from these data. Parental history of myopia was determined by asking whether the father or mother wore spectacles or contact lenses for short sightedness. The completed levels of education of the father and mother were ascertained (elementary school, high school, or tertiary education) and we asked about the number of books the child read per week.

\section{DATA ANALYSIS}

The Pearson correlation coefficient of spherical equivalent refractions from the right and left eye was 0.96. As results from the right and left eye were similar, only right eye data were presented. Biometry parameters and refraction were compared for children with parents with different smoking status and who reported different numbers of cigarettes smoked using the non-parametric tests, the Wilcoxon rank sum test, and Kruskall Wallis test. The prediction of parental smoking status on the various biometry parameters, corneal curvature, and refraction were assessed in multivariate linear regression models, adjusting for age, sex, school, parent's myopia, and parent's education. Additional models adjusting for reading as well were also constructed. All probabilities quoted are two sided and considered statistically significant when less than 0.05. A sample size of 1052 was needed for a two sided hypothesis to detect a mean refractive error of $-0.6 \mathrm{D}$ (SD 0.5) in children exposed to passive smoke compared with -0.5 (SD 0.5) for children not exposed to passive smoke, if the power (1- $\beta$ ) was 0.9 and type I error, $\alpha$, was 0.05 . Data analysis was conducted using commercially available software (STATA, version 7.0). ${ }^{11}$

\section{RESULTS}

There were 231 children aged 8 years, 561 aged 9 years, 455 aged 10 years, and 87 aged 11 years; 686 were males and 648 were females. The proportion of children who were myopic (spherical equivalent refraction at least $-0.5 \mathrm{D}$ ) was $55.2 \%$ (95\% CI 52.5 to 57.8 ). The mean axial length was $23.86 \mathrm{~mm}$ (SD 1.06; range 21.68-27.60). Of the 1324 children with maternal smoking information, $17(1.3 \%)$ of the mothers were current smokers and nine $(0.7 \%)$ were former smokers (table 1). There were 23 (1.7\%) women who smoked during the child's lifetime and the mean length of time that the mothers smoked was 7.5 years. There were only three mothers who smoked during the child's pregnancy. There were 434 fathers $(33.3 \%)$ who smoked during the child's lifetime and the mean length of time the fathers smoked was 8.5 years. There were 1295 children with both paternal and

\begin{tabular}{|c|c|c|}
\hline & Father, $\mathbf{n}(\%)$ & Mother, n $(\%)$ \\
\hline \multicolumn{3}{|c|}{ Cigarette smoking } \\
\hline Never & $816(62.4)$ & $1298(98.0)$ \\
\hline Current & $349(26.7)$ & $17(1.3)$ \\
\hline Former & $142(10.9)$ & $9(0.7)$ \\
\hline \multicolumn{3}{|c|}{$\begin{array}{l}\text { Cigarette smoking during child's } \\
\text { lifetime }\end{array}$} \\
\hline No & $871(66.7)$ & $1301(98.3)$ \\
\hline Yes & $434(33.3)$ & $23(1.7)$ \\
\hline \multicolumn{3}{|c|}{$\begin{array}{l}\text { Current smokers: } \\
\text { Number of cigarettes smoked per } \\
\text { day }\end{array}$} \\
\hline 0 & 843 (63.2) & $1308(98.1)$ \\
\hline 6 or less & 177 (13.3) & 15 (1.1) \\
\hline $7-12$ & $161(12.1)$ & $6(0.5)$ \\
\hline $13-22$ & $115(8.6)$ & $4(0.3)$ \\
\hline $23-32$ & $27(2.0)$ & $1(0.1)$ \\
\hline $33-42$ & $8(0.6)$ & $0(0.0)$ \\
\hline 43 or more & $3(0.2)$ & $0(0.0)$ \\
\hline \multicolumn{3}{|c|}{$\begin{array}{l}\text { Number of cigarettes smoked per } \\
\text { day during child's lifetime }\end{array}$} \\
\hline 0 & $871(66.8)$ & $1301(98.3)$ \\
\hline 6 or less & $152(11.7)$ & $13(1.0)$ \\
\hline $7-12$ & $142(10.9)$ & $10(0.8)$ \\
\hline $13-22$ & $106(8.1)$ & $0(0.0)$ \\
\hline $23-32$ & $23(1.8)$ & $0(0.0)$ \\
\hline $33-42$ & $8(0.6)$ & $0(0.0)$ \\
\hline 43 or more & $2(0.2)$ & $0(0.0)$ \\
\hline \multicolumn{3}{|c|}{ Duration of smoking (years) } \\
\hline Mean (SD) & $19.7(7.5)$ & $12.4(7.7)$ \\
\hline \multicolumn{3}{|c|}{$\begin{array}{l}\text { Duration of smoking during child's } \\
\text { lifetime (years) }\end{array}$} \\
\hline Mean (SD) & $8.5(2.1)$ & $7.5(3.1)$ \\
\hline
\end{tabular}

maternal smoking information. There were 417 children $(32.2 \%)$ who were exposed to passive smoke from one parent and 17 children (1.3\%) who were exposed to passive smoke from two parents. Ninety nine per cent of the fathers and $58 \%$ of the mothers were working. Of the 23 women who smoked during the child's lifetime, 11 were working $(47.8 \%)$ and $12(52.2 \%)$ were not working.

Children exposed to maternal passive smoke had refractions that shifted towards hyperopia (multivariate adjusted mean $-0.28 \mathrm{D} ; 95 \%$ CI -1.12 to +0.57$)$ compared with children whose mothers did not (multivariate adjusted mean $-1.38 \mathrm{D} ; 95 \% \mathrm{CI}-1.49$ to -1.27 ), after controlling for age, sex, school, mother's education, and mother's myopia $(\mathrm{p}=0.012)$ (table 2$)$. Mothers who smoked were less likely to have completed tertiary education $(0.0 \% \quad v \quad 11.7 \%$; $\mathrm{p}<0.001)$, less likely to have total family incomes of $>\operatorname{Sin} \$ 5,000 \quad(1 \mathrm{US} \$=1.8 \operatorname{Sin} \$) \quad(13.0 \% v 33.0 \% ; \mathrm{p}=0.004)$, less likely to live in a private house $(4.6 \% \vee 9.6 \% ; \mathrm{p}=0.63)$, and less likely to be myopic $(40.9 \% \vee 53.8 \% ; \mathrm{p}=0.23)$, although the latter two associations are not statistically significant. The axial lengths and vitreous chamber depths were shorter (adjusted means $23.34 \mathrm{~mm}$; $16.21 \mathrm{~mm}$ ) for children exposed to maternal passive smoke compared with children who were not (adjusted means $23.87 \mathrm{~mm}$; $16.77 \mathrm{~mm}$ ). The positive associations between maternal smoking and refraction or biometry remained even after additional adjustments for reading measured by books per week.

None of the assessments of paternal passive smoking was significantly associated with refractive error, axial length, or corneal curvature (table 2). After controlling for age, sex, school, father's education, and father's myopia, the adjusted mean refractive error of $-1.31 \mathrm{D}$ in children whose fathers smoked was not significantly different from refraction $(-1.37 \mathrm{D})$ in children whose fathers did not smoke $(p=0.60)$. There was no interaction between reading and 
Table 2 Ocular biometry and refraction parameters for different parental smoking history

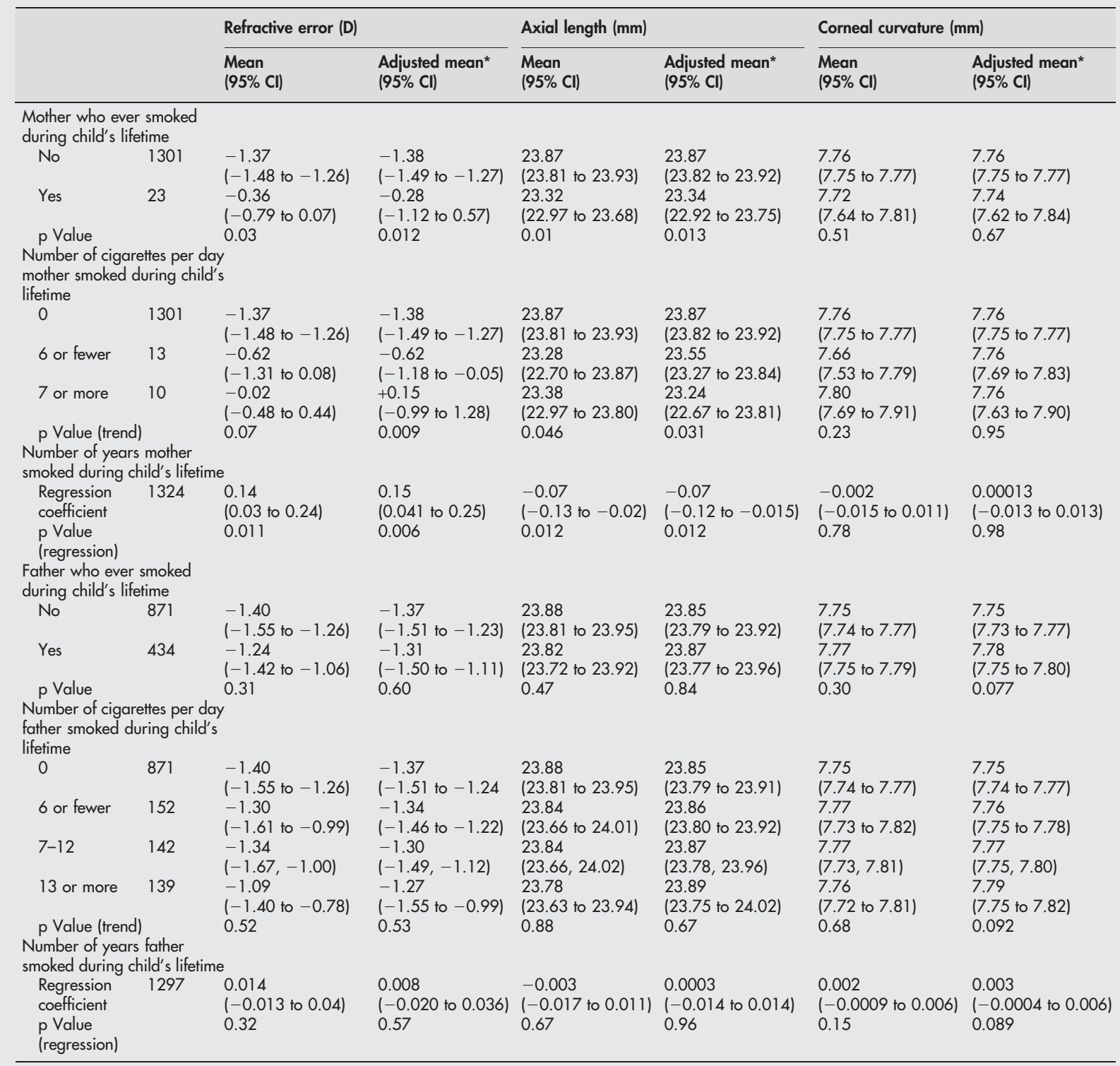

For mother's smoking, mean adjusted for age, sex, school, mother's education, and mother's myopia.

For father's smoking, mean adjusted for age, sex, school, father's education, and father's myopia.

parental smoking on refraction or any other biometry parameter.

\section{DISCUSSION}

This study sought an association between myopia in young Singapore children and parental smoking. A high prevalence and early onset of myopia is well established in this population. ${ }^{12}$ Our hypothesis was that, because of the pharmacology of nicotine, a link between myopia and passive cigarette smoking could provide pilot evidence for involvement of nicotinic cholinergic receptors in human refractive development. Although it appears that children of mothers who smoked were more likely to have hyperopic refractions, the small number of mothers $(n=23)$ and lack of association between paternal smoking and myopia argues against the hypothesis that nicotine acetylcholine receptors are the cause of a link between maternal smoking and refractive error development.

In the only pertinent previous report of which we are aware, parental smoking history was included as a risk factor for visual acuity of poorer than 0.7 in at least one eye in a study of 480 students aged 11-12 years in Japan. ${ }^{13}$ In this Japanese population, the odds ratios of having visual acuity in the better eye worse than 0.7 was 0.73 (95\% CI 0.44 to 1.22) for maternal smoking and 1.16 (95\% CI 0.72 to 1.86) for paternal smoking.

Because of these uncertainties about a potential influence of passive smoking exposure per se, a role for nicotinic acetylcholine receptors in human eye development remains indeterminate from the present results. Research in chicks has suggested that myopia is inhibited by antagonists to nicotinic acetylcholine receptors. ${ }^{7}$ From our study, however, no definite link between passive smoking and myopia was established, and there is no conclusive evidence thus far that refractive error development may be mediated through nicotine acetylcholine receptors.

Genetics and/or behaviour in families might ultimately explain why the influence of mothers and fathers might not be equal. As half the smoking mothers worked, the possibility of closer contact between mothers and young children does 
not provide a clear explanation. Among classic myopia risk factors, socioeconomic status and educational levels also do not clearly explain the association with maternal but not paternal smoking because the socioeconomic status and educational level of both parents in a nuclear family would be expected to be similar and thus influence maternal and paternal associations similarly-contrary to the actual study results. Alternatively, the lack of an effect of paternal smoking on myopia may suggest that perhaps there is no true association between passive smoking and myopia.

The major limitation of our study is the small proportion of women in Singapore who smoke $(n=23$, or $1.7 \%$ of the mothers here). Because of the small number of mothers who smoked, we cannot exclude that the association resulted from residual confounding and/or unmeasured confounders or whether it was a chance finding. A possibility is that data on other unidentified or unexplored risk factors, such as diet, academic ability, or other behavioural lifestyles, may not have been collected and their potential contributions may not be accounted for in the analyses. There may be residual confounding despite attempts to control for maternal education: children of mothers who smoked may have lower socioeconomic status, lower academic ability, prescribe to different diets, or lead different lifestyles compared with children of mothers who do not smoke.

Among other potential limitations, the reliability and quantitative accuracy of questionnaire derived data on the amount and duration of smoking is an uncertainty. As a rough validation of the questionnaire answers, the low proportion of smoking mothers generally conforms to the independently established smoking behaviour in Singapore. Specifically, the 1998 National Health Survey of 4723 Singaporean adults, aged 18-69 years, indicated that the proportions of daily smokers were $26.9 \%$ for males and $3.1 \%$ for females, and that $9.9 \%$ of males were ex-smokers and $0.7 \%$ of females were ex-smokers. ${ }^{14}$ Urinary or deciduous teeth nicotine levels were not collected because these assays are difficult from the perspective of participation. The cross sectional nature of the study also limits the ability to infer a causal relation between parental smoking and refractive errors.

Clearly, more work is needed to test the concept that passive smoking exposure might be associated with refractive development in children, and to determine whether passive smoking per se comprises the primary risk factor, an intermediate risk factor, or whether maternal smoking is a marker for other potential behavioural, social, or genetic factors that directly affect refractive development. Our data do not establish whether nicotine acetylcholine receptors may play a role in human refractive error development, as the more positive refractions found in children of smoking mothers may be attributed to a chance association or other causes, such as unmeasured socioeconomic or other lifestyle behavioural factors.

\section{Authors' affiliations}

S-M Saw, K-S Chia, Department of Community, Occupational and Family Medicine, National University of Singapore, Singapore, Republic of Singapore

J M Lindstrom, Department of Neuroscience, University of Pennsylvania School of Medicine, Philadelphia, Pennsylvania, USA

S-M Saw, D T H Tan, Singapore Eye Research Institute, Singapore and Department of Ophthalmology, National University of Singapore

R A Stone, Department of Ophthalmology, University of Pennsylvania School of Medicine, Scheie Eye Institute, Philadelphia, Pennsylvania, USA

Financial support: Funded by the National Medical Research Council, NMRC/0695/2002, NIH grants NS-1 1323 (JML) and EY-07354 (RAS), Philip Morris, Inc (JML), the Paul and Evanina Bell Mackall Foundation Trust (RAS), and a Research to Prevent Blindness Physician-Scientist Merit Award (RAS).

Disclosure of interest: JML and RAS are co-inventors on a patent application assigned to the University of Pennsylvania dealing with the use of nictonic receptor antagonists as anti-myopia agents. The other authors have no commercial conflicts of interest.

\section{REFERENCES}

1 Mutti DO, Mitchell GL, Moeschberger ML, et al. Parental myopia, near work, school achievement, and children's refractive error. Invest Ophthalmol Vis Sci 2002;43:3633-40.

2 Saw SM, Katz J, Schein OD, et al. Epidemiology of myopia. Epidemiol Rev 1996;18:175-87.

3 Wallman J, McFadden S. Monkey eyes grow into focus. Nature 1995;1:737-39.

4 Kennedy RH. Progression of myopia. Trans Am Ophthalmol Soc 1995;93:755-800.

5 Caulfield MP, Birdsall NJ. International Union of Pharmacology, XVII. Classification of muscarinic acetylcholine receptors. Pharmacol Rev 1998;50:279-90.

6 Picciotto MR, Caldarone BJ, Brunzell DH, et al. Neuronal nicotinic acetylcholine receptor subunit knockout mice: physiological and behavioural phenotypes and possible clinical implications. Pharmacol Ther 2001;92:89-108.

7 Stone RA, Sugimoto R, Gill AS, et al. Effects of nicotinic antagonists on ocular growth and experimental myopia. Invest Ophthalmol Vis Sci 2001:42:557-65.

8 Saw SM, Chua WH, Hong CY, et al. Height and its relationship to refraction and biometry parameters in Singapore Chinese children. Invest Ophthalmol Vis Sci 2002;43:1408-13.

9 Saw SM, Chua WH, Hong CY, et al. Nearwork in early-onset myopia. Invest Ophthalmol Vis Sci 2002;43:332-9.

10 Saw SM, Carkeet A, Chia KS, et al. Component dependent risk factors for ocular parameters in Singapore Chinese children. Ophthalmology 2002; 109:2065-71

11 StataCorp 2001. Stat Statistical Software: Release 7.0. College Station, TX: Stata Corporation.

12 Wu HM, Seet B, Yap EP, et al. Does education explain ethnic differences in myopia prevalence? A population-based study of young adult males in Singapore. Optom Vis Sci 2001;78:234-9.

13 Nishi M, Miyake H, Shikai T, et al. Factors influencing the visual acuity of primary school pupils. J Epidemiol 2000;10:179-82.

14 Epidemiology and Control Department, Ministry of Health, Singapore National Health Survey 1998. Singapore: Ministry of Health, 1999. 